\title{
Chaotic front dynamics in semiconductor superlattices
}

\author{
A. Amann, J. Schlesner, A. Wacker, and E. Schöll \\ Institut für Theoretische Physik, Technische Universität Berlin, Hardenbergstrasse 36, 10623, Berlin, Germany
}

(Dated: 11.12.2001)

\begin{abstract}
We analyze the dynamical evolution of the current and the charge density in a superlattice for fixed external dc voltage in the regime of self-sustained current oscillations, using a microscopic sequential tunneling model. Fronts of accumulation and depletion layers which are generated at the emitter may collide and annihilate, thereby leading to a variety of different scenarios. We find complex chaotic regimes at high voltages and low contact conductivities.
\end{abstract}

PACS numbers: 72.20.Ht,05.45.Pq,73.61.-r Keywords:

\section{INTRODUCTION}

Electronic transport in semiconductor superlattices (SL) is known to show strongly nonlinear spatio-temporal dynamics. Either self-sustained current oscillations [1, 2, 3, 4, 5] or a sawtooth-like current-voltage characteristic with many branches associated with static field domains [6, 7, 8, 9] have been found. For a recent review see [10]. Under time-dependent external voltage conditions, superlattices exhibit a rich menagerie of complex behavior including ac driven chaos and switching scenarios between multistable states. This was studied recently experimentally [11, 12, 13, 14, 15 and theoretically 16, 17.

In this paper we present simulations of dynamic scenarios for superlattices under fixed time-independent external voltage in the regime where self-sustained dipole waves are spontaneously generated at the emitter. The dipole waves are associated with traveling field domains, and consist of electron accumulation and depletion fronts which in general travel at different velocities and may merge and annihilate. We find that depending on the applied voltage and the contact conductivity, this gives rise to various oscillation modes and self-synchronization effects as well as different routes to chaotic behavior.

A similar scenario of merging fronts was recently found in the context of a spatially continuous model describing bulk impurity impact ionization breakdown 18]. It is also reminiscent of patterns of temperature pulses in globally coupled heterogeneous catalytic systems, e.g. [19].

\section{THE MODEL}

Weakly coupled superlattices are successfully described by a one dimensional sequential tunneling model for electrons 3, 20, 21. In the framework of this model electrons are assumed to be localized at one particular well and only weakly coupled to the neighboring wells. The tunneling rate to the next well is lower than the typical relaxation rate between the different energy levels within one well. The electrons within one well are then in quasi-equilibrium and transport through the barrier is incoherent. The resulting tunneling current density
$J_{m \rightarrow m+1}\left(F_{m}, n_{m}, n_{m+1}\right)$ from well $m$ to well $m+1$ depends only on the electric field $F_{m}$ between both wells and the electron densities $n_{m}$ and $n_{m+1}$ in the wells (in units of $\mathrm{cm}^{-2}$ ). For details of the microscopic calculation of $J_{m \rightarrow m+1}$ we refer to the literature [10, 17]. A typical result for the current density vs electric field characteristic is depicted in Fig. 11 in the spatially homogeneous case, i.e. $n_{m}=n_{m+1}=N_{D}$, with donor density $N_{D}$.

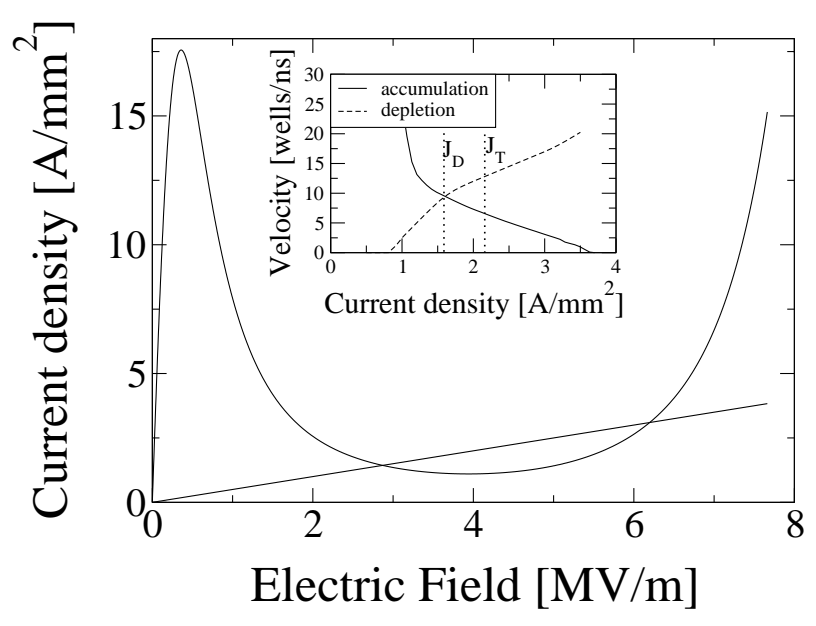

FIG. 1: Current density vs electric field characteristic at the emitter barrier (straight line) and between two neutral wells. The Ohmic conductivity of the emitter is $\sigma=0.5 \quad \Omega^{-1} m^{-1}$. The inset shows the front velocity vs. current density for electron depletion and accumulation fronts. $J_{D}$ and $J_{T}$ denote the current for dipole and tripole propagation, respectively.

In the following we will adopt the total number of electrons in one well as the dynamic variables of the system. The dynamic equations are then given by the continuity equation

$$
e \frac{\mathrm{d} n_{m}}{\mathrm{~d} t}=J_{m-1 \rightarrow m}-J_{m \rightarrow m+1} \quad \text { for } m=1, \ldots N
$$

where $N$ is the number of wells in the superlattice.

The electron densities and the electric fields are coupled by the following discrete version of Gauss's law

$$
\epsilon_{r} \epsilon_{0}\left(F_{m}-F_{m-1}\right)=e\left(n_{m}-N_{D}\right) \quad \text { for } m=1, \ldots N
$$


where $\epsilon_{r}$ and $\epsilon_{0}$ are the relative and absolute permittivities, $e<0$ is the electron charge, and $F_{0}$ and $F_{N}$ are the fields at the emitter and collector barrier, respectively.

The applied voltage between emitter and collector gives rise to a global constraint

$$
U=\sum_{m=0}^{N} F_{m} d,
$$

where $d$ is the superlattice period.

The current densities at the contacts are chosen such that dipole waves are generated at the emitter. For this purpose it is sufficient to choose Ohmic boundary conditions:

$$
\begin{aligned}
J_{0 \rightarrow 1} & =\sigma F_{0} \\
J_{N \rightarrow N+1} & =\sigma F_{N} \frac{n_{N}}{N_{D}}
\end{aligned}
$$

where $\sigma$ is the Ohmic contact conductivity, and the factor $n_{N} / N_{D}$ is introduced in order to avoid negative electron densities at the collector. Here we make the physical assumption that the current from the last well to the collector is proportional to the electron density in the last well. It is in principle possible to calculate the boundary conditions using microscopic considerations [22, 23], but the qualitative behavior is not changed.

\section{NUMERICAL RESULTS}

In our computer simulations we use an $N=100$ superlattice with $\mathrm{Al}_{0.3} \mathrm{Ga}_{0.7} \mathrm{As}$ barriers of width $b=5 \mathrm{~nm}$ and GaAs quantum wells of width $w=8 \mathrm{~nm}$, doping density $N_{D}=1.0 \times 10^{11} \mathrm{~cm}^{-2}$ and scattering induced broadening $\Gamma=8 \mathrm{meV}$ at $T=20 \mathrm{~K}$. The contact conductivity $\sigma$ is choosen such that the intersection point with the homogeneous current density vs field characteristic in Fig. 1 is at a current value at which no stationary field domain boundaries exists. By this configuration, accumulation and depletion fronts are generated at the emitter. For large values of $\sigma$, e.g. $\sigma=1.3 \quad \Omega^{-1} \mathrm{~m}^{-1}$, we find that those fronts form a dipole, i.e. a traveling field domain, with leading electron depletion front and trailing accumulation front (cf Fig. 2 (a)). The dipole traverses the sample at almost constant velocity and constant current which shows up as a plateau in the time trace of the current. The current $J_{D}$ and the front velocity in this dipole propagation mode are given by the intersection point of the velocities of accumulation and depletion front (cf. inset of Fig. 1). As the leading depletion front reaches the collector, there is no speed constraint on the remaining accumulation front. As it accelerates, the current rises, and a new dipole is generated at the emitter. Now for a short time there are two accumulation and one depletion fronts in the sample. In order to fulfill eq.(3) for fixed $U$ the depletion front has to assume twice the velocity of the accumulation fronts. This constraint fixes the current to
$J_{T}$ (cf. inset of Fig. 1) during this tripole regime. Note that in the current time trace the fast small-amplitude oscillations (due to well-to-well hopping of depletion and accumulation fronts in our discrete model) in the dipole and tripole regime are not resolved temporally.

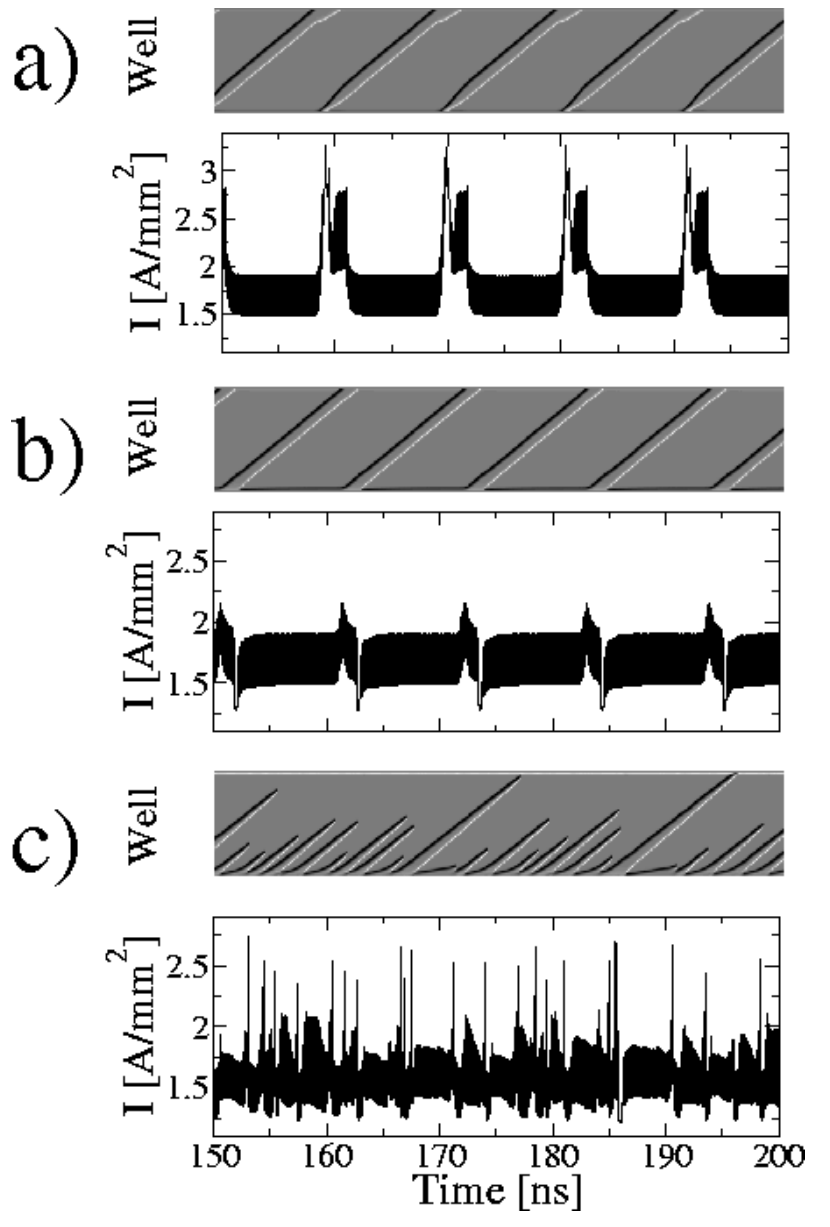

FIG. 2: Dynamic evolution of charge density and current $I$ for contact conductivities (a) $\sigma=1.3 \Omega^{-1} \mathrm{~m}^{-1}$, (b) $\sigma=$ $0.6 \Omega^{-1} \mathrm{~m}^{-1}$ and (c) $\sigma=0.55 \Omega^{-1} \mathrm{~m}^{-1}$ for fixed bias $U=1.0 \mathrm{~V}$. Light and dark regions denote electron accumulation and depletion fronts in the space-time plots of the charge densities, respectively.

At lower contact conductivity $\sigma=0.6 \quad \Omega^{-1} \mathrm{~m}^{-1}$, we find that instead of a dipole only a depletion front is generated at the emitter, as the old depletion front reaches the collector (cf. Fig. 2 (b)). Now for a short time a dipole with leading accumulation front exists. The current is fixed again by the constraint of equal velocities of accumulation and depletion front, as for the dipole with leading depletion front. At the time the old accumulation front reaches the collector a new accumulation front is generated at the emitter. This process is accompanied by a dip in the current time trace.

For even lower $\sigma=0.55 \quad \Omega^{-1} \mathrm{~m}^{-1}$ the fronts at the emitter are generated as dipoles with leading accumulation and trailing depletion fronts (cf. Fig. 2 (c)). The 

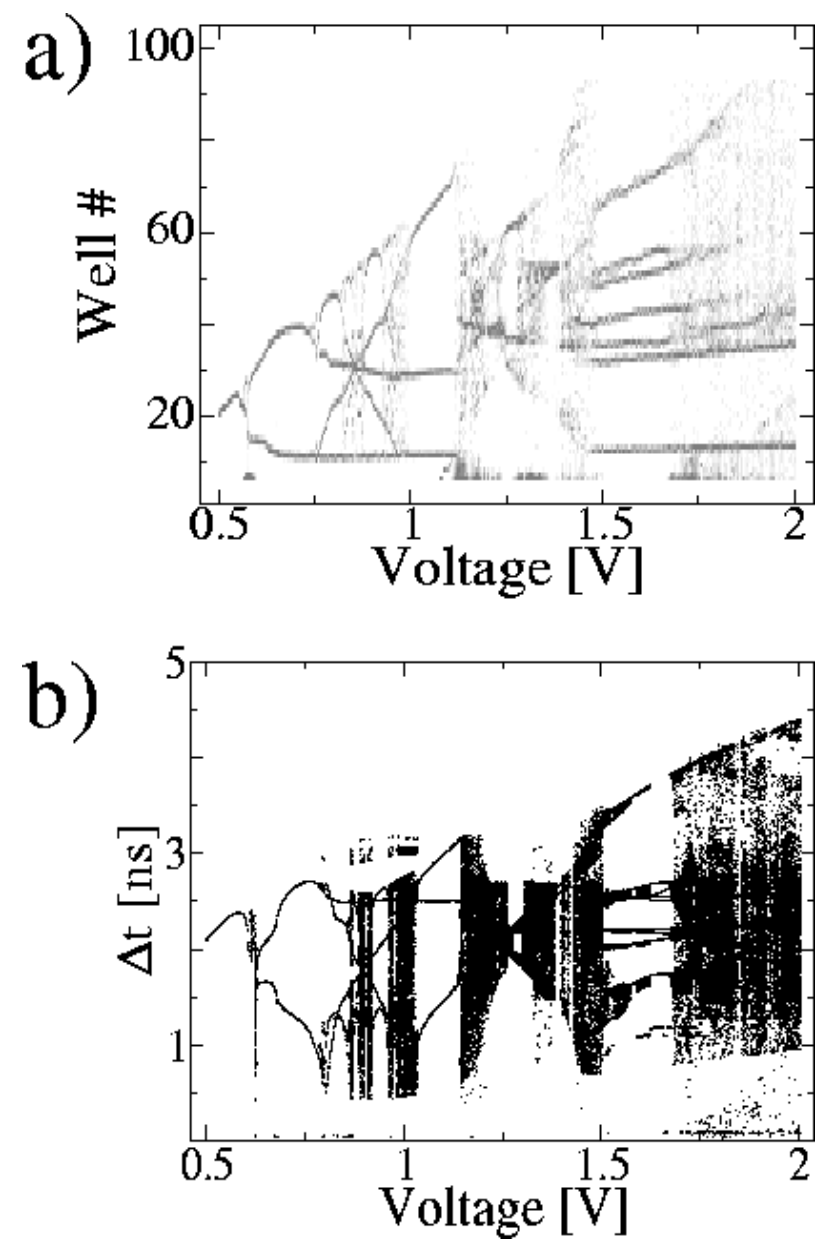

FIG. 3: (a) Positions where accumulation and depletion fronts annihilate vs voltage at $\sigma=0.5 \quad \Omega^{-1} \mathrm{~m}^{-1}$. The grayscale indicates high (black) and low (white) numbers of annihilations at a given well. (b) Time differences between consecutive maxima of the electron density in well no. 20 vs voltage at $\sigma=0.5 \Omega^{-1} \mathrm{~m}^{-1}$. Time series of length $600 \mathrm{~ns}$ have been used for each value of the voltage.

velocity of the fronts is again determined by the current and the number of fronts in the sample. Since the two types of fronts in general move at different velocities, merging and annihilation of accumulation and depletion fronts may occur, which may lead to complicated behaviour including chaos as shown in Fig. 2 (c).

In order to study the bifurcation scenario leading to chaos we now fix the boundary conductivity to $\sigma=$ $0.5 \Omega^{-1} \mathrm{~m}^{-1}$. Experimentally, low contact conductivities may be realized by changing the doping and barrier structure of the emitter. The temperature dependence of the emitter current may also be exploited. In Fig. 3 (a) a density plot of the positions (well numbers) at which two fronts annihilate is shown as a function of the voltage. We see that for low voltage the annihilation takes place at one definite position in the superlattice with a variation of only a few wells. This distribution broadens

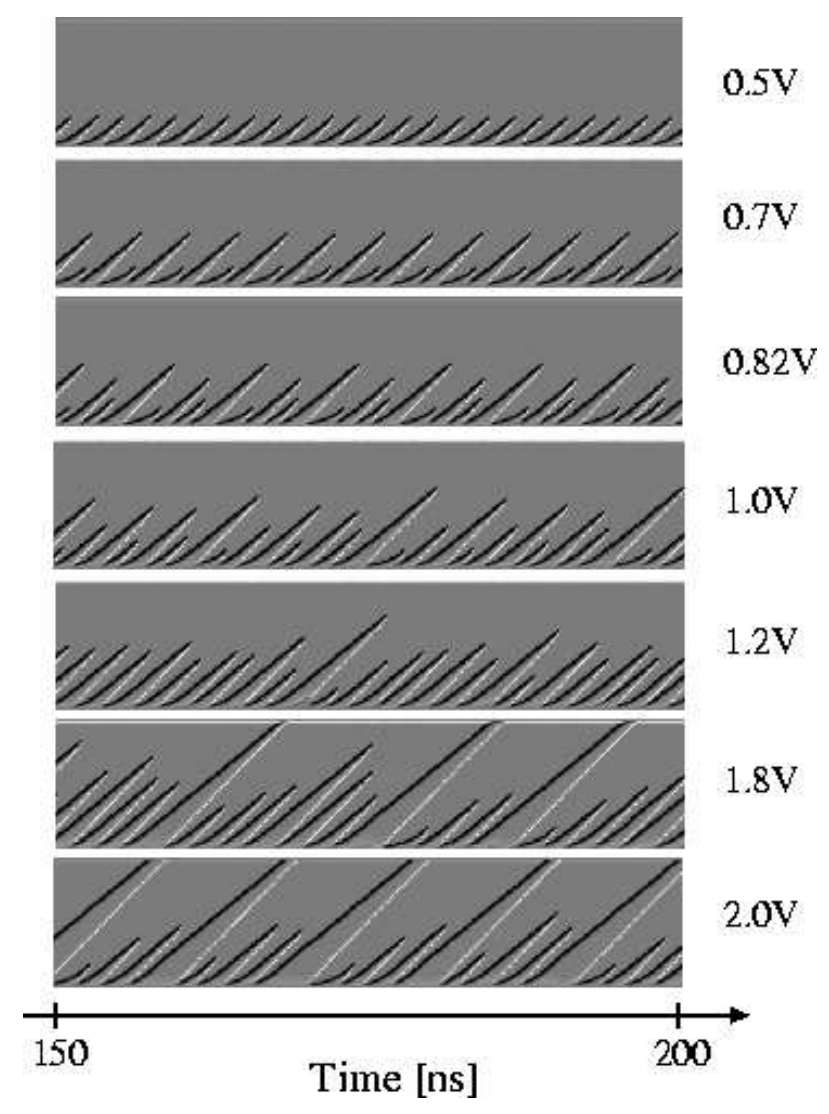

FIG. 4: Dynamic evolution of charge density for various voltages at $\sigma=0.5 \quad \Omega^{-1} \mathrm{~m}^{-1}$. Light and dark regions denote electron accumulation and depletion layer, respectively.

for increasing voltage in characteristic bifurcation scenarios reminiscent of period doubling, leading to chaotic regimes. We note that in the chaotic region periodic windows exist. Since we are dealing with a discrete system, the position of the merging of two fronts is also discrete. For more accurate analyses it is convenient to use a continuous variable such as the time difference between two maxima in the electron density in a specific well. The corresponding bifurcation diagram for well no. 20 is shown in Fig. 3 (b). It exhibits a complex structure.

The transition from periodic to chaotic oscillations is enlightened by considering the space-time plots for the evolution of the electron densities at different voltages $U$ (Fig. (1). While for $U=0.5 \mathrm{~V}$ the oscillations are regular, we see that at $U=0.7 \mathrm{~V}$ long and short front patterns alternate, which is characteristic for a period doubling bifurcation. Every second pair of fronts travels farther into the superlattice before they annihilate. At $U=0.79 \mathrm{~V}$ a further period doubling occurs, as can be most clearly seen at the alternating length of the longer front patterns. This yields a period-four cycle. Further increase of the voltage finally leads to well developed chaos, with front patterns of different lengths. For $U \geq 1.8 \mathrm{~V}$ we find that fronts may even traverse the sample, while the dynamics 
remains chaotic.

\section{SUMMARY}

We have shown that complex chaotic spatio-temporal scenarios may arise in weakly coupled superlattices under time-independent external voltage conditions, whereas previous studies have focussed on ac-driven chaos.

This work was partially supported by DFG in the framework of Sfb 555. We greatfully acknowledge discussion with L. L. Bonilla, M. Rogozia and E. Schomburg.
[1] J. Kastrup, R. Klann, H. T. Grahn, K. Ploog, L. L. Bonilla, J. Galán, M. Kindelan, M. Moscoso, and R. Merlin, Phys. Rev. B 52, 13761 (1995).

[2] K. Hofbeck, J. Grenzer, E. Schomburg, A. A. Ignatov, K. F. Renk, D. G. Pavel'ev, Y. Koschurinov, B. Melzer, S. Ivanov, S. Schaposchnikov, and P. S. Kop'ev, Phys. Lett. A 218, 349 (1996).

[3] J. Kastrup, R. Hey, K. H. Ploog, H. T. Grahn, L. L. Bonilla, M. Kindelan, M. Moscoso, A. Wacker, and J. Galán, Phys. Rev. B 55, 2476 (1997).

[4] M. Patra, G. Schwarz, and E. Schöll, Phys. Rev. B 57, 1824 (1998).

[5] H. Steuer, A. Wacker, E. Schöll, M. Ellmauer, E. Schomburg, and K. F. Renk, Appl. Phys. Lett. 76, 2059 (2000).

[6] L. Esaki and L. L. Chang, Phys. Rev. Lett. 33, 495 (1974).

[7] Y. Kawamura, K. Wakita, H. Asahi, and K. Kurumada, Jpn. J. Appl. Phys. 25, L928 (1986).

[8] H. T. Grahn, R. J. Haug, W. Müller, and K. Ploog, Phys. Rev. Lett. 67, 1618 (1991).

[9] E. Schöll, Nonlinear spatio-temporal dynamics and chaos in semiconductors (Cambridge University Press, Cambridge, 2001).

[10] A. Wacker, Phys. Rep. 357, 1 (2002).

[11] Y. Zhang, J. Kastrup, R. Klann, K. Ploog, and H. T. Grahn, Phys. Rev. Lett. 77, 3001 (1996).
[12] J. Kastrup, F. Prengel, H. T. Grahn, K. Ploog, and E. Schöll, Phys. Rev. B 53, 1502 (1996).

[13] K. J. Luo, H. T. Grahn, and K. H. Ploog, Phys. Rev. B 57, 6838 (1998).

[14] Y. Shimada and K. Hirakawa, Jpn. J. Appl. Phys. 36, 1944 (1997).

[15] M. Rogozia, S. W. Teitsworth, H. T. Grahn, and K. H. Ploog, Phys. Rev. B 64, 041398(R) (2001).

[16] O. M. Bulashenko and L. L. Bonilla, Phys. Rev. B 52, 7849 (1995).

[17] A. Amann, A. Wacker, L. L. Bonilla, and E. Schöll, Phys. Rev. E 63, 066207 (2001).

[18] I. R. Cantalapiedra, M. J. Bergmann, L. L. Bonilla, and S. W. Teitsworth, Phys. Rev. E 63, 056216 (2001).

[19] M. D. Graham, U. Middya, and D. Luss, Phys. Rev. E 48, 2917 (1993).

[20] F. Prengel, A. Wacker, and E. Schöll, Phys. Rev. B 50, 1705 (1994), ibid 52, 11518 (1995).

[21] L. L. Bonilla, J. Galán, J. A. Cuesta, F. C. Martínez, and J. M. Molera, Phys. Rev. B 50, 8644 (1994).

[22] R. Aguado, G. Platero, M. Moscoso, and L. L. Bonilla, Phys. Rev. B 55, 16053 (1997).

[23] L. L. Bonilla, G. Platero, and D. Sánchez, Phys. Rev. B 62, 2786 (2000). 